\title{
ANÁLISIS DEL PATRÓN FACIAL Y SU RELACIÓN CON LA SONRISA EN USUARIOS QUE CONCURREN A LA ORGANIZACIÓN INTERNACIONAL DE POLICÍA CRIMINAL INTERPOL PERÚ, SEDE DESCENTRALIZADA AREQUIPA, PERÚ
}

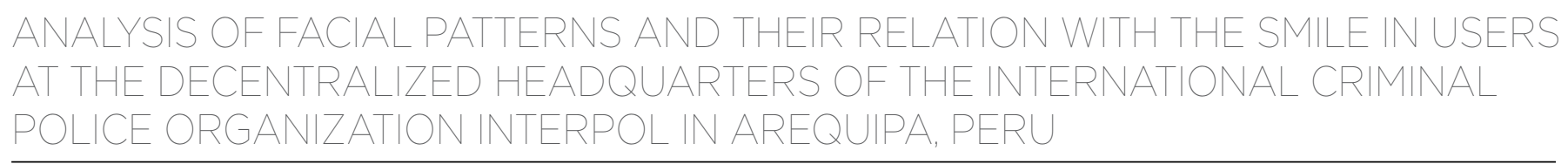

\section{Fernando Claudio Vizcarra-Zorrilla 1 * (i)} ferchocvz@gmail.com
Artículo recibido: 10/04/2020

Arbitrado por pares

Artículo aceptado: 18/06/2020

Artículo publicado: 24/08/2020

\section{* Autor corresponsal:}

Fernando Claudio Vizcarra-Zorrilla ferchocvz@gmail.com
Citar como: Vizcarra-Zorrilla F. Análisis del patrón facial y su relación con la sonrisa en usuarios que concurren a la organización internacional de policía criminal Interpol Perú, sede descentralizada Arequipa (Arequipa, Perú). Rev Cient Odontol (Lima). 2020; 8 (2): e016.

DOI: $10.21142 / 2523-2754-0802-2020-016$

\section{RESUMEN}

Objetivo: Analizar el patrón facial y su relación con la sonrisa en usuarios que acudieron a la Organización Internacional de Policía Criminal Interpol - Perú, Sede Descentralizada Arequipa, en 2018. Materiales y métodos: Se escogió una muestra de 72 usuarios de entre 20 y 40 años, de quienes se obtuvo el índice facial morfológico y se determinó el patrón facial. Se tomó 3 fotografías en sonrisa posada para realizar el análisis de los componentes de la sonrisa: corredor bucal, plano frontal oclusal, arco de sonrisa, línea labial, curvatura del labio superior; simetría de la sonrisa y componentes dentarios y gingivales. Los datos fueron analizados mediante la prueba exacta de Fisher o prueba de chi cuadrado para establecer la asociación de las variables. Resultados: El patrón facial euriprosopo presenta una línea labial baja $(50 \%)$ y media $(45,8 \%)$, los patrones faciales mesoprosopo y leptoprosopo presentan una línea labial media (54,2\% y 50,0\% respectivamente); los patrones faciales euriprosopo y mesoprosopo presentan un plano frontal oclusal no aceptable (58,3\% y 54,2\%, respectivamente), y el patrón facial leptoprosopo presenta un plano frontal oclusal aceptable (58,3\%). Los demás componentes de la sonrisa son similares en los tres patrones faciales. Conclusiones: Los resultados obtenidos establecen que no existe asociación entre los patrones faciales y los componentes de la sonrisa, puesto que no hubo valores estadísticamente significativos ( $p>0,05)$. Por lo tanto, no hay suficiente evidencia para concluir que las variables están asociadas.

Palabras clave: patrón facial, índice facial morfológico, sonrisa posada

\begin{abstract}
Aim: To analyze facial patterns and their relationship with the smile in users at the decentralized headquarters of the International Criminal Police Organization INTERPOL in Arequipa, Peru in 2018. Materials and methods: A sample of 72 individuals between 20 and 40 years old was selected, in whom the facial morphological index was obtained, and the facial pattern was determined. Three posed smile photographs were taken to analyze the smile components: oral corridor, frontal occlusal plane, smile arch, lip line, curvature of the upper lip; symmetry of the smile and dental and gingival components. The data were analyzed using the Fisher's exact test or Chi square test to establish associations among variables. Results: The euryprosopo facial pattern presents a low smile line (50\%) and average smile line (45.8\%), while mesoprosopo and leptoprosopo facial patterns present an average smile line (54.2\% and 50.0\% respectively). Euryprosopo and mesoprosopo facial patterns have an unacceptable occlusal frontal plane (58.3\% and $54.2 \%$ respectively), and the leptoprosopo facial pattern has an acceptable occlusal frontal plane (58.3\%). The remaining smile components are similar among the three facial patterns. Conclusions: The results obtained establish that there is no association between facial patterns and smile components. In the absence of statistically significant values $(p>0.05)$, there is not enough evidence to conclude that the variables are associated.
\end{abstract}

Keywords: facial pattern, morphological facial index, posed smile

1 División de Rehabilitación Oral, Carrera de Estomatología de la Universidad Científica del Sur. Lima, Perú. 


\section{INTRODUCCIÓN}

La sonrisa constituye la más notoria característica de la estética facial. Está considerada como una valiosa forma de comunicación social no verbal y un aspecto preponderante para la belleza facial. El atractivo facial y la belleza de la sonrisa guardan estrecha relación, pues hacen de la expresión facial y la apariencia un reflejo de la sonrisa, con lo que esta se convierte en un componente dominante de la estética facial $\left({ }^{1,2}\right)$.

Tanto la sonrisa agradable como la atractiva expresión facial proporcionan seguridad a la persona, lo que le permite relacionarse mejor con su entorno laboral y personal. La sonrisa y el espectro amplio de emociones que una persona puede expresar, a menudo, puede determinar qué tan bien funciona una persona en la sociedad $\left({ }^{3}\right)$. En el trabajo clínico diario, los pacientes que vienen aquejados por alguna dolencia manifiestan la necesidad de aliviar su malestar y, como deseo importante, mejorar su estética con la finalidad de verse y sentirse mejor. Esta percepción de mejoría les devuelve la seguridad comprometida por la dolencia.

La sonrisa se puede clasificar como posada (social), la cual no va acompañada de ninguna emoción, pues se trata de una expresión facial voluntaria, estática, no entrenada y repetible; y sonrisa espontánea o de placer (no posada), que se acompaña de una auténtica emoción, es involuntaria, dinámica y utiliza frecuentemente todos los músculos del rostro. Se utiliza rutinariamente la sonrisa posada para evaluar la estética facial y la sonrisa, debido a que es reproducible y puede formarse después de la indicación de sonreír (4-6).

Cuando se comienza un tratamiento de odontología estética, se deben considerar las expectativas del paciente, sus características anatómicas individuales y las posibles soluciones terapéuticas $\left({ }^{7}\right)$. Una sonrisa agradable depende del componente dentario en cuanto a la posición, el color, el tamaño y la forma, así como del componente gingival respecto de la cantidad de encía expuesta y la adecuada adaptación de los labios $\left({ }^{8,9}\right)$.

Los biotipos faciales son tres: braquifacial, mesofacial y dolicofacial. El rostro y la sonrisa deben guardar armonía para lograr el atractivo facial, y tanto la armonía como el equilibrio del perfil varían según los diferentes tipos faciales $\left({ }^{10,11}\right)$.

Cada tipo facial le da al paciente diferentes características, de acuerdo con el predominio vertical u horizontal de las dimensiones en el rostro. Los pacientes con patrón braquifacial (euriprosopo) suelen tener caras cortas y anchas, arcadas dentarias amplias con una posición más horizontal del incisivo central superior, y una distancia horizontal mayor que la vertical. Los pacientes con patrón mesofacial (mesoprosopo) presentan maxilares y arcadas dentarias ovoides proporcionales, existe equilibrio entre la distancia horizontal y vertical, y tienen una musculatura armónica. Los pacientes con patrón dolicofacial (leptoprosopo) suelen presentar la cara más alargada y arcadas dentarias estrechas, con una posición más vertical del incisivo central superior; la distancia vertical es mayor a la horizontal; poseen musculatura débil y los labios están generalmente tensos por el aumento en la altura facial inferior $\left({ }^{12}\right)$.

Los planes de tratamiento que diariamente diseñan los clínicos para devolver la función y estética perdidas en los pacientes toman como referencia estándares y biotipos de poblaciones distintas, sin considerar las características propias de la muy variada mezcla de razas, lo que produce no solo una enorme gama de rasgos faciales, sino también una combinación cultural realmente significativa.

Comprender la estrecha relación entre las estructuras orales de soporte, los músculos, los huesos, las articulaciones, el componente dentario y gingival, y la oclusión nos lleva a obtener un procedimiento rehabilitador exitoso tanto en lo funcional como en lo estético $\left({ }^{13}\right)$. El análisis de la sonrisa es una etapa importante para el diagnóstico, planificar el tratamiento y precisar el pronóstico del tratamiento rehabilitador que implique objetivos funcionales y estéticos $(2,14)$.

Evaluar los componentes de la sonrisa relacionados con el plano oclusal frontal, curvatura del labio superior, componentes dentarios, línea labial, simetría de sonrisa, componentes gingivales, arco de sonrisa y corredor bucal y vincularlos al patrón facial de cada paciente, debe ser un procedimiento rutinario simple y práctico en la consulta. 
Usamos criterios subjetivos con mucha frecuencia para evaluar la belleza de las personas y el atractivo de la sonrisa; sin embargo, la sonrisa tiene componentes objetivos que podemos utilizar en el trabajo clínico diario para un análisis que nos permita planificar el tratamiento rehabilitador y prever un pronóstico más preciso, por lo que la identificación del patrón facial y el análisis y evaluación objetiva de los componentes de la sonrisa debe ser la piedra angular del diseño de la sonrisa.

El objetivo de la siguiente investigación fue analizar el patrón facial y su relación con la sonrisa en usuarios que acudieron a la Organización Internacional de Policía Criminal Interpol Perú, sede descentralizada Arequipa, en 2018.

\section{MATERIALES Y MÉTODOS}

La muestra fue seleccionada de usuarios que se atendieron en la Organización Internacional de Policía Criminal Interpol Perú, Sede Descentralizada Arequipa, durante los meses de octubre a diciembre del 2018, para obtener la Ficha de Canje Internacional, para lo cual firmaron el consentimiento informado correspondiente.

El tamaño de la muestra, inicialmente, se calculó considerando los resultados obtenidos de un estudio piloto preliminar sobre un grupo de 22 usuarios, para lo cual se realizó la toma de medidas faciales a cada uno de ellos con el fin de establecer el patrón facial y, sobre la fotografía, determinar la presencia o ausencia del corredor bucal (componente de la sonrisa más estudiado), mediante la comparación de dos proporciones. Para ello, se utilizó el software Fisterra, y se consignó un nivel de confianza o seguridad del $95 \%$, un poder estadístico del $80 \%$, una proporción del grupo control del 33\% y una proporción en el grupo de intervención del 69\%. E1 mínimo de tamaño muestral fue de 23 sujetos por grupo y 27 sujetos tomando en consideración la proporción esperada de pérdidas (15\%).

El cálculo de la muestra brindó resultados que se han tomado como referencia para el presente estudio, y determinaron al final la muestra en relación a los artículos base que han originado este trabajo.
Los criterios de inclusión fueron los siguientes: usuarios de 20 a 40 años, de ambos sexos, con piezas dentarias anteriores completas y sanas, con buen estado de salud general. Los criterios de exclusión comprendieron usuarios con tratamiento de ortodoncia anterior o durante la evaluación, dientes anterosuperiores con restauraciones visibles, pacientes con clase II o III esquelética, personas con deformidades faciales o historia de cirugía ortognática, o con estado de salud comprometido.

Toma de medidas faciales en los usuarios. El aspecto facial, tan importante para el diagnóstico, comprendió el examen de la cara, para lo cual se realizó la toma de las medidas faciales en cada uno de los sujetos de la muestra. El patrón o tipo facial se obtuvo mediante el índice facial morfológico (figura 1), que clasifica a las personas en 3 tipos faciales: euriprosopo, mesoprosopo y leptoprosopo $\left({ }^{15}\right)$. Se determinó el tipo facial tomando la medida del punto ofrion (On) al punto gnation $(\mathrm{Gn})$, multiplicado por 100 y dividido después por la distancia bicigomática $(\mathrm{Zy}-\mathrm{Zy})$. El ofrion $(\mathrm{On})$ es el punto de intersección de la línea media sagital y una línea recta hipotética que une el borde superior de las cejas. El gnation (Gn) es el punto más anterior e inferior del mentón sobre el plano medio sagital. El zygion $(\mathrm{Zy})$ es el punto más lateral de cada arco cigomático. De acuerdo con el

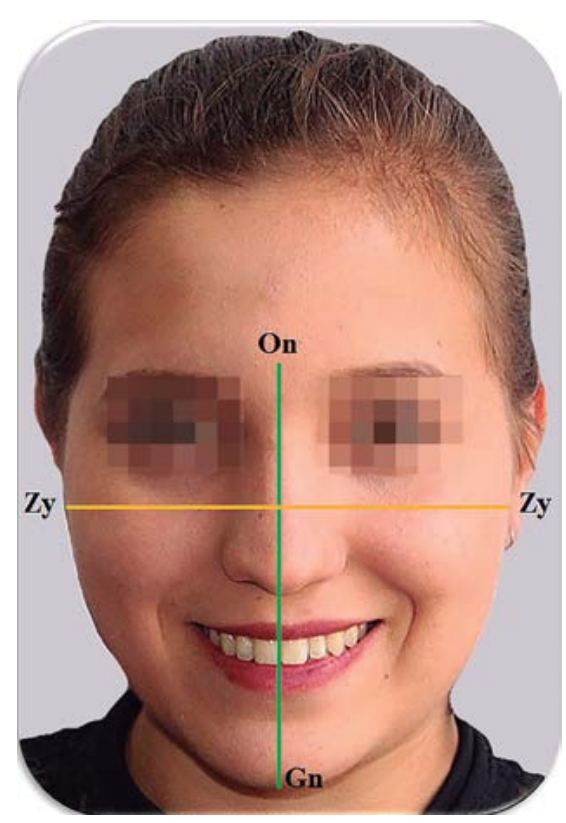

Figura I. Índice facial morfológico 


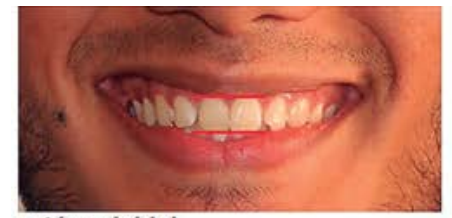

a. Línea labial

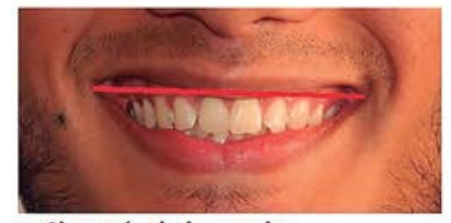

e. Simetría de la sonrisa

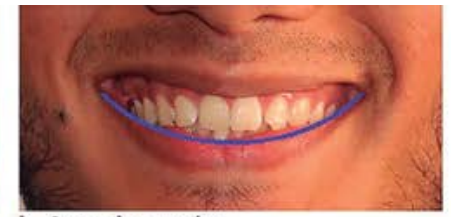

b. Arco de sonrisa

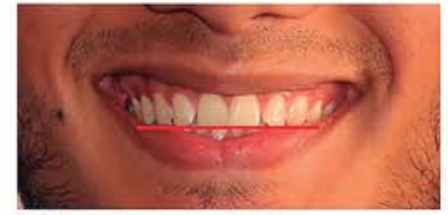

f. Plano frontal oclusal

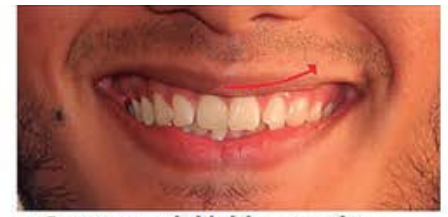

c. Curvatura del labio superior

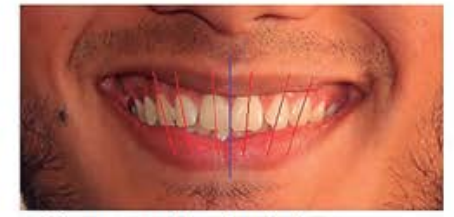

g. Componentes dentarios

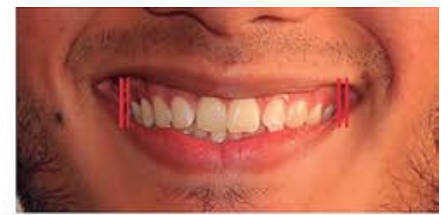

d. Corredores bucales

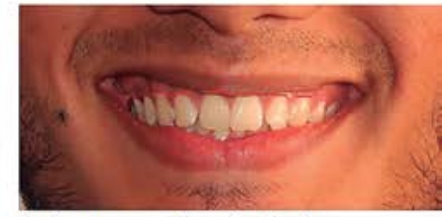

h. Componentes gingivales

Figura 2. Componentes de la sonrisa

valor obtenido, se pueden distinguir los siguientes tipos faciales: euriprosopo (braquifacial), cuando los valores obtenidos son menores a 97; mesoprosopo (mesofacial), cuando los valores oscilen entre 97 y 104; y leptoprosopo (dolicofacial), cuando los valores son superiores a $104\left({ }^{15}\right)$.

Toma de fotografías. Se tomó 3 fotografías en sonrisa posada del usuario, utilizando una cámara fotográfica semiprofesional Canon EOS T5 Rebel 18-55 mm, con una intensidad de luz entre 5500 y 6000 grados Kelvin para reproducir el color preciso de la piel. Cada sujeto de la muestra se ubicó sobre una silla separada $30 \mathrm{~cm}$ de la pared y a $100 \mathrm{~cm}$ de la cámara fotográfica, mirando hacia el frente, directamente al lente, y manteniendo la línea interpupilar horizontal. Los sujetos no usaron accesorios como lentes, aretes o piercing que dificulten el análisis facial, y el cabello se recogió detrás de las orejas. De las tomas obtenidas se eligió la más natural posible.

Determinación del tipo de sonrisa. Los componentes de la sonrisa (figura 2) que se utilizaron fueron los empleados por Sabri $\left({ }^{16}\right)$ :

a. Línea labial. Se midió el labio superior con relación a los incisivos centrales superiores. Se consideró alta cuando el labio superior mostró todo el largo de los incisivos centrales y una línea ininterrumpida de encía, media cuando el labio superior muestra todo el largo de los incisivos centrales superiores y la papila interdentaria, y baja cuando el labio superior muestra el $75 \%$ o menos de todo el largo de los incisivos centrales superiores. b. Arco de sonrisa. Se midió una curva trazada a lo largo de los bordes incisales de los dientes anterosuperiores en sonrisa. Se consideró consonante cuando la curvatura de los incisivos superiores era paralela al contorno interno del labio inferior al sonreír y no consonante cuando la curvatura de los incisivos superiores estaba aplanada o inversa en comparación con el labio inferior al sonreír.

c. Curvatura del labio superior. Se midió la disposición del tubérculo central del labio superior respecto de la comisura labial al sonreír. Se consideró alta cuando la comisura labial era más alta que la ubicación del tubérculo central, recta cuando la comisura labial y el tubérculo central estaban a la misma altura, y baja cuando la comisura labial aparecía en una posición más baja que el tubérculo central.

d. Corredor bucal. Se midió el corredor bucal a nivel de los dientes posteriores y la comisura labial. Se consideró presente cuando existió el corredor bucal y ausente cuando no existió.

e. Simetría de la sonrisa. Se midió la ubicación de las comisuras labiales con relación al plano vertical. Se consideró simétrica cuando ambas comisuras labiales se encontraban a la misma altura y asimétrica cuando las comisuras labiales no se encontraban a la misma altura.

f. Plano oclusal frontal. Se trazó una línea hipotética entre las puntas de ambos caninos superiores. Se 
consideró aceptable cuando la línea trazada era paralela a la línea bipupilar, y no aceptable cuando la línea trazada no era paralela a esta.

g. Componentes dentarios. Se midieron los componentes dentarios respecto de su tamaño, color, forma, alineación, coincidencia de las líneas media facial y media dentaria, y simetría del arco dentario. Se consideró aceptable cuando existía simetría dentaria entre ambos lados de la boca, simetría de $\operatorname{arcos} y$ paralelismo y coincidencia de las líneas media facial y media dentaria, y no aceptable cuando no existía simetría dentaria de arcos o ausencia de paralelismo y coincidencia de las líneas media facial y media dentaria.

h. Componentes gingivales. Se midió los componentes gingivales con relación a la altura, textura, color y contorno de la encía. Se consideró aceptable cuando existía simetría gingival (márgenes gingivales parejos) entre ambos lados de la boca, sin signos de inflamación o espacios gingivales abiertos, y no aceptable cuando no existía simetría gingival (márgenes gingivales parejos) entre ambos lados de la boca, y aparecían signos de inflamación o espacios gingivales abiertos.

Se utilizó el programa estadístico SPSS Versión 22 (IBM SPSS, Chicago, Illinois, EE. UU.) para el procesamiento y análisis estadístico de datos. Se empleó la prueba exacta de Fisher o la prueba de chi cuadrado para establecer la asociación de las variables cualitativas causa (patrón facial) y efecto (componentes de la sonrisa) utilizadas en este estudio. Se ha elegido el valor de $\mathrm{p}<0,05$ para todas las pruebas.

\section{RESULTADOS}

La muestra del estudio estuvo conformada por 72 personas, 43 (59,7\%) de sexo femenino y 29 (40,3\%) de sexo masculino. En el caso del sexo femenino, la edad promedio fue de 26 años y de 31 años en el caso del sexo masculino, mientras que en ambos la edad mínima fue de 20 años y la edad máxima, de 39 años, con una desviación típica de 4,817 para el sexo femenino y 4,706 para el masculino (tabla 1 ).
En el caso del sexo femenino, la línea labial que predominó en el tipo facial euriprosopo fue la baja $(50,0 \%)$, seguida por la media $(44,4 \%)$. Esta última predominó en el tipo facial mesoprosopo, con el 52,9\%. Las líneas labiales que predominaron en el tipo facial leptoprosopo fueron la baja y la alta, con el 37,55\% cada una. En el caso del sexo masculino, las líneas labiales baja y media predominaron en el tipo facial euriprosopo, con valores del 50,0\% cada una. La línea labial media predominó en los tipos faciales mesoprosopo y leptoprosopo, con el 57,1\% y el 62,5\%, respectivamente. La línea labial que predominó en general en el tipo facial euriprosopo fue la baja $(50,0 \%)$, seguida por la media $(45,8 \%)$; mientras que en los tipos faciales mesoprosopo y leptoprosopo la línea labial media fue la predominante, con el 54,2\% y el 50,0\%, respectivamente (tabla 2). No se presentó asociación estadísticamente significativa, $\mathrm{p}=0,199(>0,05)$.

El arco de sonrisa no consonante predominó en el sexo femenino en los tipos faciales euriprosopo y mesoprosopo, con el 55,6\% y el 58,8\%, respectivamente. En el tipo facial leptoprosopo, el arco de sonrisa tiene valores similares consonante y no consonante de 50,0\% cada uno. En el sexo masculino, los tipos faciales euriprosopo, mesoprosopo y leptoprosopo, mostraron un arco de sonrisa no consonante con $83,3 \%, 100 \%$ y $87,5 \%$ respectivamente. El arco de la sonrisa no consonante predominó, en general, en los tipos faciales euriprosopo, mesoprosopo y leptoprosopo, con el 62,5\%, 70,8\% y 75,0\%, respectivamente (tabla 3). No se presentó asociación estadísticamente significativa $\mathrm{p}=0,731(>0,05)$.

La curvatura del labio superior recta predominó en el sexo femenino en los tipos faciales euriprosopo, mesoprosopo y leptoprosopo, con el 55,6\%, 76,5\% y $87,5 \%$, respectivamente. La curvatura del labio superior

Tabla 1. Distribución de frecuencia según sexo y edad en usuarios que acuden a la Interpol Perú, Sede Descentralizada Arequipa

\begin{tabular}{lccccc} 
& \multicolumn{4}{c}{ Edad } \\
Sexo del usuario & $\mathbf{n}$ & Mínimo & Máximo & Media & Desv. típ. \\
\hline Femenino & 43 & 20 & 39 & 26,44 & 4,817 \\
\hline Masculino & 29 & 20 & 39 & 31,00 & 4,706 \\
\hline
\end{tabular}


Tabla 2. Distribución de frecuencia del patrón facial según la línea labial y el sexo en usuarios que acuden a la Interpol Perú, Sede Descentralizada Arequipa

\begin{tabular}{|c|c|c|c|c|c|c|c|c|}
\hline \multirow{2}{*}{\multicolumn{3}{|c|}{ Sexo del participante }} & & \multicolumn{3}{|c|}{ Patrón facial } & \multirow{2}{*}{ Total } & \multirow{2}{*}{$\mathbf{X} 2$} \\
\hline & & & & Euriprosopo & Mesoprosopo & Leptoprosopo & & \\
\hline \multirow{8}{*}{ Femenino } & \multirow{6}{*}{ Línea labial } & \multirow{2}{*}{ Baja } & $n$ & 9 & 4 & 3 & 16 & \\
\hline & & & $\%$ & $50,0 \%$ & $23,5 \%$ & $37,5 \%$ & $37,2 \%$ & \\
\hline & & \multirow{2}{*}{ Media } & $n$ & 8 & 9 & 2 & 19 & \\
\hline & & & $\%$ & $44,4 \%$ & $52,9 \%$ & $25,0 \%$ & $44,2 \%$ & \\
\hline & & \multirow{2}{*}{ Alta } & $n$ & । & 4 & 3 & 8 & \\
\hline & & & $\%$ & $5,6 \%$ & $23,5 \%$ & $37,5 \%$ & $18,6 \%$ & \\
\hline & \multirow{2}{*}{ Total } & & $n$ & 18 & 17 & 8 & 43 & \\
\hline & & & $\%$ & $100,0 \%$ & $100,0 \%$ & $100,0 \%$ & $100,0 \%$ & \\
\hline \multirow{8}{*}{ Masculino } & \multirow{6}{*}{ Línea labial } & \multirow{2}{*}{ Baja } & $n$ & 3 & 3 & 3 & 9 & \\
\hline & & & $\%$ & $50,0 \%$ & $42,9 \%$ & $18,8 \%$ & $31,0 \%$ & \\
\hline & & \multirow{2}{*}{ Media } & $n$ & 3 & 4 & 10 & 17 & \\
\hline & & & $\%$ & $50,0 \%$ & $57,1 \%$ & $62,5 \%$ & $58,6 \%$ & \\
\hline & & \multirow{2}{*}{ Alta } & $n$ & 0 & 0 & 3 & 3 & \\
\hline & & & $\%$ & $0,0 \%$ & $0,0 \%$ & $18,8 \%$ & $10,3 \%$ & \\
\hline & \multirow{2}{*}{ Total } & & $n$ & 6 & 7 & 16 & 29 & \\
\hline & & & $\%$ & $100,0 \%$ & $100,0 \%$ & $100,0 \%$ & $100,0 \%$ & \\
\hline \multirow{8}{*}{ Total } & \multirow{6}{*}{ Línea labial } & \multirow{2}{*}{ Baja } & $n$ & 12 & 7 & 6 & 25 & \\
\hline & & & $\%$ & $50,0 \%$ & $29,2 \%$ & $25,0 \%$ & $34,7 \%$ & \\
\hline & & \multirow{2}{*}{ Media } & $n$ & 11 & 13 & 12 & 36 & \\
\hline & & & $\%$ & $45,8 \%$ & $54,2 \%$ & $50,0 \%$ & $50,0 \%$ & \\
\hline & & \multirow{2}{*}{ Alta } & $n$ & I & 4 & 6 & II & \\
\hline & & & $\%$ & $4,2 \%$ & $16,7 \%$ & $25,0 \%$ & |5,3\% & \\
\hline & \multirow{2}{*}{\multicolumn{2}{|c|}{ Total }} & $n$ & 24 & 24 & 24 & 72 & \multirow{2}{*}{$p=0,199$} \\
\hline & & & $\%$ & $100,0 \%$ & $100,0 \%$ & $100,0 \%$ & $100,0 \%$ & \\
\hline
\end{tabular}

baja predominó en el sexo masculino en los tipos faciales mesoprosopo y leptoprosopo, con el $71,4 \%$ y el $56,3 \%$, respectivamente; mientras que la curvatura del labio superior baja y recta fue predominante en el tipo facial euriprosopo, con valores similares del 50,0\%. La curvatura del labio superior recta ha predominado en general en los tipos faciales euriprosopo, mesoprosopo y leptoprosopo, con valores del 54,2\%, 58,3\% y $54,2 \%$, respectivamente (tabla 4). No hubo asociación estadísticamente significativa $\mathrm{p}=0,425(>0,05)$.

El corredor bucal presente ha predominado en el sexo femenino en los tipos faciales euriprosopo, mesoprosopo y leptoprosopo, con el 66,7\%, 76,5\% y 75,0\%, respectivamente. En el sexo masculino, el corredor bucal predominante en los tipos faciales euriprosopo, mesoprosopo y leptoprosopo fue también el presente, con el $83,3 \%, 57,1 \%$ y $56,3 \%$, respectivamente. La presencia del corredor bucal en los tipos faciales euriprosopo, mesoprosopo y leptoprosopo fue significativa, con valores del 70,8\%, 70,8\% y 62,5\%, respectivamente (tabla 5). No se presentó asociación estadísticamente significativa $\mathrm{p}=0,856(>0,05)$.

La sonrisa simétrica predomina en el sexo femenino en los tipos faciales euriprosopo, mesoprosopo y leptoprosopo, con el 66,7\%,70,6\% y 75,0\%, respectivamente. La sonrisa simétrica predomina en el sexo masculino en los patrones faciales euriprosopo y leptoprosopo, con valores del $83,3 \%$ y el 56,3\%, respectivamente. La sonrisa asimétrica ha predominado en el patrón facial mesoprosopo, con un valor del $57,1 \%$. La sonrisa simétrica predomina en general en los tipos faciales euriprosopo, mesoprosopo y leptoprosopo, con valores del 70,8\%, 62,5\% y 62,5\%, 
Tabla 3. Distribución de frecuencia del patrón facial según el arco de la sonrisa y el sexo en usuarios que acuden a la Interpol Perú, Sede Descentralizada Arequipa

\begin{tabular}{|c|c|c|c|c|c|c|c|c|}
\hline \multirow{2}{*}{\multicolumn{2}{|c|}{ Sexo del participante }} & & & \multicolumn{3}{|c|}{ Patrón facial } & \multirow{2}{*}{ Total } & \multirow{2}{*}{$\mathbf{X}_{2}$} \\
\hline & & & & Euriprosopo & Mesoprosopo & Leptoprosopo & & \\
\hline \multirow{6}{*}{ Femenino } & \multirow{4}{*}{$\begin{array}{l}\text { Arco de la } \\
\text { sonrisa }\end{array}$} & \multirow{2}{*}{ Consonante } & $n$ & 8 & 7 & 4 & 19 & \\
\hline & & & $\%$ & $44,4 \%$ & $41,2 \%$ & $50,0 \%$ & $44,2 \%$ & \\
\hline & & \multirow{4}{*}{ No consonante } & $n$ & 10 & 10 & 4 & 24 & \\
\hline & & & $\%$ & $55,6 \%$ & $58,8 \%$ & $50,0 \%$ & $55,8 \%$ & \\
\hline & \multirow{2}{*}{ Total } & & $n$ & 18 & 17 & 8 & 43 & \\
\hline & & & $\%$ & $100,0 \%$ & $100,0 \%$ & $100,0 \%$ & $100,0 \%$ & \\
\hline \multirow{6}{*}{ Masculino } & \multirow{4}{*}{$\begin{array}{l}\text { Arco de la } \\
\text { sonrisa }\end{array}$} & \multirow{2}{*}{ Consonante } & $n$ & I & 0 & 2 & 3 & \\
\hline & & & $\%$ & $16,7 \%$ & $0,0 \%$ & $12,5 \%$ & $10,3 \%$ & \\
\hline & & \multirow{4}{*}{ No consonante } & $\mathrm{n}$ & 5 & 7 & 14 & 26 & \\
\hline & & & $\%$ & $83,3 \%$ & $100,0 \%$ & $87,5 \%$ & $89,7 \%$ & \\
\hline & \multirow{2}{*}{ Total } & & $n$ & 6 & 7 & 16 & 29 & \\
\hline & & & $\%$ & $100,0 \%$ & $100,0 \%$ & $100,0 \%$ & $100,0 \%$ & \\
\hline \multirow{6}{*}{ Total } & \multirow{4}{*}{$\begin{array}{l}\text { Arco de la } \\
\text { sonrisa }\end{array}$} & \multirow{2}{*}{ Consonante } & $\mathrm{n}$ & 9 & 7 & 6 & 22 & \\
\hline & & & $\%$ & $37,5 \%$ & $29,2 \%$ & $25,0 \%$ & $30,6 \%$ & \\
\hline & & \multirow{4}{*}{ No consonante } & $n$ & 15 & 17 & 18 & 50 & \\
\hline & & & $\%$ & $62,5 \%$ & $70,8 \%$ & $75,0 \%$ & $69,4 \%$ & \\
\hline & \multirow{2}{*}{ Total } & & $n$ & 24 & 24 & 24 & 72 & \multirow{2}{*}{$p=0,731$} \\
\hline & & & $\%$ & $100,0 \%$ & $100,0 \%$ & $100,0 \%$ & $100,0 \%$ & \\
\hline
\end{tabular}

respectivamente (tabla 6). No se presentó asociación estadísticamente significativa $\mathrm{p}=0,861(>0,05)$.

El plano frontal oclusal predominante en el sexo femenino en los tipos faciales euriprosopo y mesoprosopo fue el no aceptable, con el 55,6\% y el 52,9\%, respectivamente. El plano frontal oclusal predominante en el tipo facial leptoprosopo fue el aceptable, con el 62,5\%. El plano frontal oclusal predominante en el sexo masculino en los tipos faciales euriprosopo y mesoprosopo fue el no aceptable, con el $66,7 \%$ y el $57,1 \%$, respectivamente. El plano frontal oclusal predominante en el tipo facial leptoprosopo fue el aceptable, con el 56,3\%. El plano frontal oclusal no aceptable ha predominado en general en los tipos faciales euriprosopo y mesoprosopo, con valores del 58,3\% y el 54,2\%, respectivamente. El plano frontal oclusal que ha predominado en el patrón facial leptoprosopo fue el aceptable, con el 58,3\% (tabla 7). No se presentó asociación estadísticamente significativa $\mathrm{p}=0,580(>0,05)$.

El componente dentario predominante en el sexo femenino en los tipos faciales euriprosopo y mesoprosopo fue el no aceptable, con el 77,8\% y el $64,7 \%$, respectivamente. E1 componente dentario en el tipo facial leptoprosopo tiene valores similares del $50,0 \%$ para aceptable y no aceptable. El componente dentario predominante en el sexo masculino en los tipos faciales euriprosopo, mesoprosopo y leptoprosopo fue el no aceptable, con valores del 83,3\%, 85,7\% y 81,3\%, respectivamente. El componente dentario no aceptable ha predominado en general en los tipos faciales euriprosopo, mesoprosopo y leptoprosopo, con valores del 79,2\%, 70,8\% y 70,8\%, respectivamente (tabla 8 ). No se presentó asociación estadísticamente significativa $\mathrm{p}=0,840(>0,05)$.

El componente gingival no aceptable ha predominado en el sexo femenino en el tipo facial euriprosopo, con el $72,2 \%$. El componente gingival aceptable ha predominado en el tipo facial mesoprosopo, con el $52,9 \%$, seguido muy de cerca por el componente gingival no aceptable, con el 47,1\%. El componente gingival en el tipo facial leptoprosopo en el sexo femenino tiene valores similares del 50,0\% para aceptable y no aceptable. $\mathrm{El}$ componente gingival no aceptable ha predominado 
Tabla 4. Distribución de frecuencia del patrón facial según la curvatura del labio superior y el sexo en usuarios que acuden a la Interpol Perú, Sede Descentralizada Arequipa

\begin{tabular}{|c|c|c|c|c|c|c|c|c|}
\hline \multicolumn{2}{|c|}{ Sexo del participante } & & & \multicolumn{3}{|c|}{ Patrón facial } & Total & $\mathbf{X} 2$ \\
\hline \multirow{8}{*}{ Femenino } & \multirow{6}{*}{$\begin{array}{l}\text { Curvatura del } \\
\text { labio superior }\end{array}$} & \multirow{2}{*}{ Baja } & $n$ & 3 & 3 & 1 & 7 & \\
\hline & & & $\%$ & $16,7 \%$ & $17,6 \%$ & $12,5 \%$ & $16,3 \%$ & \\
\hline & & \multirow{2}{*}{ Recta } & $n$ & 10 & 13 & 7 & 30 & \\
\hline & & & $\%$ & $55,6 \%$ & $76,5 \%$ & $87,5 \%$ & $69,8 \%$ & \\
\hline & & \multirow{2}{*}{ Alta } & $n$ & 5 & I & 0 & 6 & \\
\hline & & & $\%$ & $27,8 \%$ & $5,9 \%$ & $0,0 \%$ & $14,0 \%$ & \\
\hline & \multirow{2}{*}{ Total } & & $n$ & 18 & 17 & 8 & 43 & \\
\hline & & & $\%$ & $100,0 \%$ & $100,0 \%$ & $100,0 \%$ & $100,0 \%$ & \\
\hline \multirow{8}{*}{ Masculino } & \multirow{6}{*}{$\begin{array}{l}\text { Curvatura del } \\
\text { labio superior }\end{array}$} & \multirow{2}{*}{ Baja } & $n$ & 3 & 5 & 9 & 17 & \\
\hline & & & $\%$ & $50,0 \%$ & $71,4 \%$ & $56,3 \%$ & $58,6 \%$ & \\
\hline & & \multirow{2}{*}{ Recta } & $n$ & 3 & I & 6 & 10 & \\
\hline & & & $\%$ & $50,0 \%$ & $4,3 \%$ & $37,5 \%$ & $34,5 \%$ & \\
\hline & & \multirow{2}{*}{ Alta } & $n$ & 0 & I & I & 2 & \\
\hline & & & $\%$ & $0,0 \%$ & |4,3\% & $6,3 \%$ & $6,9 \%$ & \\
\hline & \multirow{2}{*}{ Total } & & $n$ & 6 & 7 & 16 & 29 & \\
\hline & & & $\%$ & $100,0 \%$ & $100,0 \%$ & $100,0 \%$ & $100,0 \%$ & \\
\hline \multirow{8}{*}{ Total } & \multirow{6}{*}{$\begin{array}{l}\text { Curvatura del } \\
\text { labio superior }\end{array}$} & \multirow{2}{*}{ Baja } & $n$ & 6 & 8 & 10 & 24 & \\
\hline & & & $\%$ & $25,0 \%$ & $33,3 \%$ & $41,7 \%$ & $33,3 \%$ & \\
\hline & & \multirow{2}{*}{ Recta } & $n$ & 13 & 14 & 13 & 40 & \\
\hline & & & $\%$ & $54,2 \%$ & $58,3 \%$ & $54,2 \%$ & $55,6 \%$ & \\
\hline & & \multirow{2}{*}{ Alta } & $n$ & 5 & 2 & I & 8 & \\
\hline & & & $\%$ & $20,8 \%$ & $8,3 \%$ & $4,2 \%$ & $\mid 1,1 \%$ & \\
\hline & \multirow{2}{*}{ Total } & & $n$ & 24 & 24 & 24 & 72 & \multirow{2}{*}{$p=0,425$} \\
\hline & & & $\%$ & $100,0 \%$ & $100,0 \%$ & $100,0 \%$ & $100,0 \%$ & \\
\hline
\end{tabular}

en el sexo masculino en los tipos faciales euriprosopo, mesoprosopo y leptoprosopo, con el $66,7 \%, 85,6 \%$ y $75,0 \%$, respectivamente. El componente gingival predominante en general en los tipos faciales euriprosopo, mesoprosopo y leptoprosopo fue el no aceptable, con valores del 70,8\%, 58,3\% y 66,7\%, respectivamente (tabla 9). No se presentó asociación estadísticamente significativa $\mathrm{p}=0,745(>0,05)$.

\section{DIscusión}

El presente trabajo de investigación tuvo como objetivo analizar el patrón facial y establecer su asociación con los componentes de la sonrisa. Los resultados obtenidos establecen que no existe asociación entre los patrones faciales y los componentes de la sonrisa, puesto que no se presentaron valores estadísticamente significativos $(\mathrm{p}>0,05)$.

El patrón facial euriprosopo estudiado en la muestra presenta, predominantemente, arco de sonrisa no consonante, el plano frontal oclusal no aceptable, corredores bucales presentes, curvatura del labio superior recta, líneas labiales baja y media, y sonrisa simétrica, con componentes dentario y gingival no aceptables.

El patrón facial mesoprosopo presenta, de forma predominante, curvatura del labio superior recta, arco de sonrisa no consonante, corredores bucales presentes, línea labial media, plano frontal oclusal no aceptable y sonrisa simétrica, con componentes dentario y gingival no aceptables. 
Tabla 5. Distribución de frecuencia del patrón facial según el corredor bucal y el sexo en usuarios que acuden a la Interpol Perú, Sede Descentralizada Arequipa

\begin{tabular}{|c|c|c|c|c|c|c|c|c|}
\hline \multicolumn{2}{|c|}{ Sexo del participante } & & & \multicolumn{3}{|c|}{ Patrón facial } & Total & $\mathbf{X}_{2}$ \\
\hline \multirow{6}{*}{ Femenino } & \multirow{4}{*}{ Corredor bucal } & \multirow{2}{*}{ Presente } & $n$ & 12 & 13 & 6 & 31 & \\
\hline & & & $\%$ & $66,7 \%$ & $76,5 \%$ & $75,0 \%$ & $72,1 \%$ & \\
\hline & & \multirow{4}{*}{ Ausente } & $n$ & 6 & 4 & 2 & 12 & \\
\hline & & & $\%$ & $33,3 \%$ & $23,5 \%$ & $25,0 \%$ & $27,9 \%$ & \\
\hline & \multirow{2}{*}{ Total } & & $n$ & 18 & 17 & 8 & 43 & \\
\hline & & & $\%$ & $100,0 \%$ & $100,0 \%$ & $100,0 \%$ & $100,0 \%$ & \\
\hline \multirow{6}{*}{ Masculino } & \multirow{4}{*}{ Corredor bucal } & \multirow{2}{*}{ Presente } & $n$ & 5 & 4 & 9 & 18 & \\
\hline & & & $\%$ & $83,3 \%$ & $57,1 \%$ & $56,3 \%$ & $62,1 \%$ & \\
\hline & & \multirow{4}{*}{ Ausente } & $n$ & I & 3 & 7 & 11 & \\
\hline & & & $\%$ & $16,7 \%$ & $42,9 \%$ & $43,8 \%$ & $37,9 \%$ & \\
\hline & \multirow{2}{*}{ Total } & & $n$ & 6 & 7 & 16 & 29 & \\
\hline & & & $\%$ & $100,0 \%$ & $100,0 \%$ & $100,0 \%$ & $100,0 \%$ & \\
\hline \multirow{6}{*}{ Total } & \multirow{4}{*}{ Corredor bucal } & \multirow{2}{*}{ Presente } & $n$ & 17 & 17 & 15 & 49 & \\
\hline & & & $\%$ & $70,8 \%$ & $70,8 \%$ & $62,5 \%$ & $68,1 \%$ & \\
\hline & & \multirow{4}{*}{ Ausente } & n & 7 & 7 & 9 & 23 & \\
\hline & & & $\%$ & $29,2 \%$ & $29,2 \%$ & $37,5 \%$ & $31,9 \%$ & \\
\hline & \multirow{2}{*}{ Total } & & $n$ & 24 & 24 & 24 & 72 & \multirow{2}{*}{$p=0,856$} \\
\hline & & & $\%$ & $100,0 \%$ & $100,0 \%$ & $100,0 \%$ & $100,0 \%$ & \\
\hline
\end{tabular}

Tabla 6. Distribución de frecuencia del patrón facial según la simetría de la sonrisa y el sexo en usuarios que acuden a la Interpol Perú, Sede Descentralizada Arequipa

\begin{tabular}{|c|c|c|c|c|c|c|c|c|}
\hline \multicolumn{2}{|c|}{ Sexo del participante } & & & \multicolumn{3}{|c|}{ Patrón facial } & \multirow{2}{*}{ Total } & \multirow{2}{*}{$x_{2}$} \\
\hline \multirow{7}{*}{ Femenino } & \multirow{5}{*}{$\begin{array}{l}\text { Simetría de la } \\
\text { sonrisa }\end{array}$} & \multirow{3}{*}{ Simétrica } & & & & & & \\
\hline & & & $n$ & 12 & 12 & 6 & 30 & \\
\hline & & & $\%$ & $66,7 \%$ & $70,6 \%$ & $75,0 \%$ & $69,8 \%$ & \\
\hline & & \multirow{4}{*}{ Asimétrica } & $n$ & 6 & 5 & 2 & 13 & \\
\hline & & & $\%$ & $33,3 \%$ & $29,4 \%$ & $25,0 \%$ & $30,2 \%$ & \\
\hline & \multirow{2}{*}{ Total } & & $n$ & 18 & 17 & 8 & 43 & \\
\hline & & & $\%$ & $100,0 \%$ & $100,0 \%$ & $100,0 \%$ & $100,0 \%$ & \\
\hline \multirow{6}{*}{ Masculino } & \multirow{4}{*}{$\begin{array}{l}\text { Simetría de la } \\
\text { sonrisa }\end{array}$} & \multirow{2}{*}{ Simétrica } & $n$ & 5 & 3 & 9 & 17 & \\
\hline & & & $\%$ & $83,3 \%$ & $42,9 \%$ & $56,3 \%$ & $58,6 \%$ & \\
\hline & & \multirow{4}{*}{ Asimétrica } & $n$ & 1 & 4 & 7 & 12 & \\
\hline & & & $\%$ & $16,7 \%$ & $57,1 \%$ & $43,8 \%$ & $41,4 \%$ & \\
\hline & \multirow{2}{*}{ Total } & & $\mathrm{n}$ & 6 & 7 & 16 & 29 & \\
\hline & & & $\%$ & $100,0 \%$ & $100,0 \%$ & $100,0 \%$ & $100,0 \%$ & \\
\hline \multirow{6}{*}{ Total } & \multirow{4}{*}{$\begin{array}{l}\text { Simetría de la } \\
\text { sonrisa }\end{array}$} & \multirow{2}{*}{ Simétrica } & $n$ & 17 & 15 & 15 & 47 & \\
\hline & & & $\%$ & $70,8 \%$ & $62,5 \%$ & $62,5 \%$ & $65,3 \%$ & \\
\hline & & \multirow{4}{*}{ Asimétrica } & $n$ & 7 & 9 & 9 & 25 & \\
\hline & & & $\%$ & $29,2 \%$ & $37,5 \%$ & $37,5 \%$ & $34,7 \%$ & \\
\hline & \multirow{2}{*}{ Total } & & $n$ & 24 & 24 & 24 & 72 & \multirow{2}{*}{$p=0,861$} \\
\hline & & & $\%$ & $100,0 \%$ & $100,0 \%$ & $100,0 \%$ & $100,0 \%$ & \\
\hline
\end{tabular}


Tabla 7. Distribución de frecuencia del patrón facial según el plano frontal oclusal y el sexo en usuarios que acuden a la Interpol Perú, Sede Descentralizada Arequipa

\begin{tabular}{|c|c|c|c|c|c|c|c|c|}
\hline \multicolumn{2}{|c|}{ Sexo del participante } & & & \multicolumn{3}{|c|}{ Patrón facial } & Total & $x_{2}$ \\
\hline \multirow{6}{*}{ Femenino } & \multirow{4}{*}{$\begin{array}{l}\text { Plano frontal } \\
\text { oclusal }\end{array}$} & \multirow{2}{*}{ Aceptable } & $\mathrm{n}$ & 8 & 8 & 5 & 21 & \\
\hline & & & $\%$ & $44,4 \%$ & $47,1 \%$ & $62,5 \%$ & $48,8 \%$ & \\
\hline & & \multirow{4}{*}{ No aceptable } & $\mathrm{n}$ & 10 & 9 & 3 & 22 & \\
\hline & & & $\%$ & $55,6 \%$ & $52,9 \%$ & $37,5 \%$ & $51,2 \%$ & \\
\hline & \multirow{2}{*}{ Total } & & $\mathrm{n}$ & 18 & 17 & 8 & 43 & \\
\hline & & & $\%$ & $100,0 \%$ & $100,0 \%$ & $100,0 \%$ & $100,0 \%$ & \\
\hline \multirow{6}{*}{ Masculino } & \multirow{4}{*}{$\begin{array}{l}\text { Plano frontal } \\
\text { oclusal }\end{array}$} & \multirow{2}{*}{ Aceptable } & $\mathrm{n}$ & 2 & 3 & 9 & 14 & \\
\hline & & & $\%$ & $33,3 \%$ & $42,9 \%$ & $56,3 \%$ & $48,3 \%$ & \\
\hline & & \multirow{4}{*}{ No aceptable } & $\mathrm{n}$ & 4 & 4 & 7 & 15 & \\
\hline & & & $\%$ & $66,7 \%$ & $57,1 \%$ & $43,8 \%$ & $51,7 \%$ & \\
\hline & \multirow{2}{*}{ Total } & & $\mathrm{n}$ & 6 & 7 & 16 & 29 & \\
\hline & & & $\%$ & $100,0 \%$ & $100,0 \%$ & $100,0 \%$ & $100,0 \%$ & \\
\hline \multirow{6}{*}{ Total } & \multirow{4}{*}{$\begin{array}{l}\text { Plano frontal } \\
\text { oclusal }\end{array}$} & \multirow{2}{*}{ Aceptable } & $\mathrm{n}$ & 10 & 11 & 14 & 35 & \\
\hline & & & $\%$ & $41,7 \%$ & $45,8 \%$ & $58,3 \%$ & $48,6 \%$ & \\
\hline & & \multirow{2}{*}{ No aceptable } & $n$ & 14 & 13 & 10 & 37 & \\
\hline & & & $\%$ & $58,3 \%$ & $54,2 \%$ & $41,7 \%$ & $51,4 \%$ & \\
\hline & \multirow{2}{*}{ Total } & & $\mathrm{n}$ & 24 & 24 & 24 & 72 & \multirow{2}{*}{$p=0,580$} \\
\hline & & & $\%$ & $100,0 \%$ & $100,0 \%$ & $100,0 \%$ & $100,0 \%$ & \\
\hline
\end{tabular}

Tabla 8. Distribución de frecuencia del patrón facial según el componente dentario y el sexo en usuarios que acuden a la Interpol Perú, Sede Descentralizada Arequipa

\begin{tabular}{|c|c|c|c|c|c|c|c|c|}
\hline \multicolumn{2}{|c|}{ Sexo del participante } & & & \multicolumn{3}{|c|}{ Patrón facial } & \multirow{2}{*}{ Total } & \multirow{2}{*}{$\mathbf{X} 2$} \\
\hline \multirow{7}{*}{ Femenino } & \multirow{5}{*}{$\begin{array}{l}\text { Componente } \\
\text { dentario }\end{array}$} & \multirow{3}{*}{ Aceptable } & & & & & & \\
\hline & & & $n$ & 4 & 6 & 4 & 14 & \\
\hline & & & $\%$ & $22,2 \%$ & $35,3 \%$ & $50,0 \%$ & $32,6 \%$ & \\
\hline & & \multirow{4}{*}{ No aceptable } & $n$ & 14 & 11 & 4 & 29 & \\
\hline & & & $\%$ & $77,8 \%$ & $64,7 \%$ & $50,0 \%$ & $67,4 \%$ & \\
\hline & \multirow{2}{*}{ Total } & & $n$ & 18 & 17 & 8 & 43 & \\
\hline & & & $\%$ & $100,0 \%$ & $100,0 \%$ & $100,0 \%$ & $100,0 \%$ & \\
\hline \multirow{6}{*}{ Masculino } & \multirow{4}{*}{$\begin{array}{l}\text { Componente } \\
\text { dentario }\end{array}$} & \multirow{2}{*}{ Aceptable } & $n$ & I & I & 3 & 5 & \\
\hline & & & $\%$ & $16,7 \%$ & $14,3 \%$ & $18,8 \%$ & $17,2 \%$ & \\
\hline & & \multirow{4}{*}{ No aceptable } & $n$ & 5 & 6 & 13 & 24 & \\
\hline & & & $\%$ & $83,3 \%$ & $85,7 \%$ & $81,3 \%$ & $82,8 \%$ & \\
\hline & \multirow{2}{*}{ Total } & & $\mathrm{n}$ & 6 & 7 & 16 & 29 & \\
\hline & & & $\%$ & $100,0 \%$ & $100,0 \%$ & $100,0 \%$ & $100,0 \%$ & \\
\hline \multirow{6}{*}{ Total } & \multirow{4}{*}{$\begin{array}{l}\text { Componente } \\
\text { dentario }\end{array}$} & \multirow{2}{*}{ Aceptable } & $n$ & 5 & 7 & 7 & 19 & \\
\hline & & & $\%$ & $20,8 \%$ & $29,2 \%$ & $29,2 \%$ & $26,4 \%$ & \\
\hline & & \multirow{4}{*}{ No aceptable } & $n$ & 19 & 17 & 17 & 53 & \\
\hline & & & $\%$ & $79,2 \%$ & $70,8 \%$ & $70,8 \%$ & $73,6 \%$ & \\
\hline & \multirow{2}{*}{ Total } & & $n$ & 24 & 24 & 24 & 72 & \multirow{2}{*}{$p=0,84 C$} \\
\hline & & & $\%$ & $100,0 \%$ & $100,0 \%$ & $100,0 \%$ & $100,0 \%$ & \\
\hline
\end{tabular}


Tabla 9. Distribución de frecuencia del patrón facial según el componente gingival y el sexo en usuarios que acuden a la Interpol Perú, Sede Descentralizada Arequipa

\begin{tabular}{|c|c|c|c|c|c|c|c|c|}
\hline \multirow{2}{*}{\multicolumn{2}{|c|}{ Sexo del participante }} & & & \multicolumn{3}{|c|}{ Patrón facial } & \multirow{2}{*}{ Total } & \multirow{2}{*}{$x_{2}$} \\
\hline & & & & Euriprosopo & Mesoprosopo & Leptoprosopo & & \\
\hline \multirow{6}{*}{ Femenino } & \multirow{4}{*}{$\begin{array}{l}\text { Componente } \\
\text { gingival }\end{array}$} & \multirow{2}{*}{ Aceptable } & $n$ & 5 & 9 & 4 & 18 & \\
\hline & & & $\%$ & $27,8 \%$ & $52,9 \%$ & $50,0 \%$ & $41,9 \%$ & \\
\hline & & \multirow{4}{*}{ No aceptable } & $\mathrm{n}$ & 13 & 8 & 4 & 25 & \\
\hline & & & $\%$ & $72,2 \%$ & $47,1 \%$ & $50,0 \%$ & $58,1 \%$ & \\
\hline & \multirow{2}{*}{ Total } & & $n$ & 18 & 17 & 8 & 43 & \\
\hline & & & $\%$ & $100,0 \%$ & $100,0 \%$ & $100,0 \%$ & $100,0 \%$ & \\
\hline \multirow{6}{*}{ Masculino } & \multirow{4}{*}{$\begin{array}{l}\text { Componente } \\
\text { gingival }\end{array}$} & \multirow{2}{*}{ Aceptable } & $\mathrm{n}$ & 2 & I & 4 & 7 & \\
\hline & & & $\%$ & $33,3 \%$ & $14,3 \%$ & $25,0 \%$ & $24,1 \%$ & \\
\hline & & \multirow{4}{*}{ No aceptable } & $\mathrm{n}$ & 4 & 6 & 12 & 22 & \\
\hline & & & $\%$ & $66,7 \%$ & $85,7 \%$ & $75,0 \%$ & $75,9 \%$ & \\
\hline & \multirow{2}{*}{ Total } & & $n$ & 6 & 7 & 16 & 29 & \\
\hline & & & $\%$ & $100,0 \%$ & $100,0 \%$ & $100,0 \%$ & $100,0 \%$ & \\
\hline \multirow{6}{*}{ Total } & \multirow{4}{*}{$\begin{array}{l}\text { Componente } \\
\text { gingival }\end{array}$} & \multirow{2}{*}{ Aceptable } & $\mathrm{n}$ & 7 & 10 & 8 & 25 & \\
\hline & & & $\%$ & $29,2 \%$ & $41,7 \%$ & $33,3 \%$ & $34,7 \%$ & \\
\hline & & \multirow{4}{*}{ No aceptable } & $\mathrm{n}$ & 17 & 14 & 16 & 47 & \\
\hline & & & $\%$ & $70,8 \%$ & $58,3 \%$ & $66,7 \%$ & $65,3 \%$ & \\
\hline & \multirow{2}{*}{ Total } & & $n$ & 24 & 24 & 24 & 72 & \multirow{2}{*}{$p=0,745$} \\
\hline & & & $\%$ & $100,0 \%$ & $100,0 \%$ & $100,0 \%$ & $100,0 \%$ & \\
\hline
\end{tabular}

El patrón facial leptoprosopo presenta, predominantemente, curvatura del labio superior recta, arco de sonrisa no consonante, línea labial media, corredores bucales presentes, plano frontal oclusal aceptable y sonrisa simétrica, con componentes dentario y gingival no aceptables.

Posada et al. $\left({ }^{17}\right)$ describieron la relación existente entre la línea de la sonrisa y la forma del rostro en una población de Medellín (Colombia), y encontraron que, en los tipos faciales leptoprosopo, mesoprosopo y euriprosopo, predominaba la línea de sonrisa media, lo que coincide en parte con los resultados de esta investigación, dado que en los tipos faciales mesoprosopo y leptoprosopo destaca la línea labial media en un porcentaje del $54 \%$ y el 50\%, respectivamente, lo que no ocurre en el euriprosopo, que presenta sobre todo una línea de sonrisa baja (50\%). Es importante hacer notar que el estudio de Posada ha manejado un intervalo de edad de 18 a 77 años, por lo que la línea labial puede variar como consecuencia de la atrición y la abrasión producto de la edad.
Un estudio realizado por Tjan et al. $\left(^{18}\right)$ encontró, en un grupo de pacientes americanos blancos, una línea de sonrisa media $(68,94 \%)$ y la curva incisal superior paralela al borde interno del labio inferior $(84,8 \%)$. Este resultado es contrario al nuestro, que arrojó un arco de sonrisa no consonante. Es necesario mencionar que el estudio no hizo referencia alguna al patrón facial.

Los resultados obtenidos en un estudio hecho por Cruz et al. $\left({ }^{10}\right)$ en ciudadanos adultos mexicanos proporcionan información respecto del biotipo facial predominante (euriprosopo, 82,5\%), arco de sonrisa consonante y corredores bucales presentes en los biotipos euriprosopo, mesoprosopo y leptoprosopo. Esto coincide con nuestros resultados respecto de los corredores bucales presentes en las muestras, pero difiere en el arco de sonrisa no consonante.

Zhang et al. $\left({ }^{19}\right)$ evaluaron la sonrisa dinámica en jóvenes chinos y hallaron que el 45,5\% tenía una línea labial alta y el mismo porcentaje una línea labial media, mientras 
que las jóvenes chinas mostraban mayor cantidad de encía. Ellos no relacionan su estudio con el patrón facial; sin embargo, hacen referencia a un estudio previo sobre la prevalencia de la forma braquicefálica de la cabeza en adultos asiáticos $\left({ }^{20}\right)$.

Mahn et al. $\left({ }^{9}\right)$ evaluaron las sonrisas posada y dinámica en una población estudiantil joven (18 a 32 años) de la Universidad de los Andes de Chile, y encontraron que la línea labial alta fue la más frecuente en la sonrisa espontánea, mientras que en la posada lo fue la línea labial media, lo cual coincide en parte con los resultados de una línea labial media en los tipos faciales mesoprosopo y leptoprosopo, y una línea labial baja y media en el tipo euriprosopo.

En su investigación, Köseoğlu et al. ( $\left.{ }^{21}\right)$ evaluaron el ancho del corredor bucal, el arco de la sonrisa, el tipo de sonrisa y la línea de sonrisa en jóvenes turcos de 18 a 25 años, y hallaron que la sonrisa comisural es la más común en ambos géneros, mientras que la menos común es la sonrisa compleja. La línea de sonrisa media es la predominante y la alta, la menos común, y esto varía según el género. El tipo de línea incisal más recurrente es la línea convexa, en ambos sexos, y el menos común es la línea incisal inversa. El corredor bucal presente se registró en toda la muestra. No hizo referencia alguna al patrón facial.

Krishnan et al. $\left.{ }^{6}\right)$ apreciaron las características de la sonrisa mediante el análisis de fotografías frontales de la sonrisa posada en personas indias. Identificaron más sonrisas consonantes en mujeres que en hombres y no encontraron diferencias significativas en la medición de los corredores bucales entre ambos sexos. Las comparaciones entre los índices faciales y las características de la sonrisa no mostraron relación alguna.

Siddiqui et al. $\left({ }^{22}\right)$ evaluaron la sonrisa dinámica en distintos patrones esqueléticos y concluyeron que los diferentes patrones esqueléticos presentan sonrisas dinámicas distintivas: el patrón esquelético horizontal presenta un arco de sonrisa plana y el patrón esquelético vertical, un arco de sonrisa paralelo, con mayor frecuencia.
La belleza del rostro en general, y de la sonrisa en particular, es hoy en día el objetivo primordial para muchos pacientes, realidad a la que se añade el reconocimiento de que las características corporales varían de una persona a otra debido a la mezcla de razas y las características propias de cada cultura.

Es primordial reconocer las diferencias étnicas para lograr una armonía estética adecuada en los pacientes de un grupo racial en particular, y tener en cuenta que las comunidades multirraciales crean combinaciones de características únicas $\left.{ }^{23}\right)$.

\section{CONCLUSIONES}

- El patrón facial euriprosopo presenta predominantemente una línea labial baja y media, mientras que los tipos mesoprosopo y leptoprosopo presentan una línea labial media.

- Los patrones faciales euriprosopo y mesoprosopo presentan un plano frontal oclusal no aceptable; por su parte, el patrón facial leptoprosopo presenta un plano frontal oclusal aceptable.

- Los tres patrones faciales (euriprosopo, mesoprosopo y leptoprosopo) presentan características similares en los componentes de la sonrisa: el arco de la sonrisa no consonante, la curvatura del labio superior recta, los espacios laterales negativos presentes, la sonrisa simétrica, los componentes dentarios y los componentes gingivales no aceptables.

- Es primordial determinar las características de la sonrisa y el tipo facial empleando componentes objetivos sencillos de medir, las particularidades de las diferentes etnias, así como las singularidades de cada paciente y su propia percepción respecto del atractivo y armonía facial, así como de la sonrisa, para establecer el diagnóstico pertinente y un adecuado plan de tratamiento. 
Contribución de autoría: Fernando Claudio Vizcarra Zorrilla ha concebido el trabajo, recopilado la información, llevado a cabo el análisis y la interpretación de los datos, así como la redacción y la aprobación de la versión final del artículo.

\section{REFERENCIAS BIBLIOGRÁFICAS}

1. Padrós E. Bases diagnósticas, terapéuticas y posturales del funcionalismo craneofacial. Madrid: Ripano; 2006.

2. Machado AW. 10 commandments of smile esthetics. Dental Press J Orthod. 2014; 19 (4): 136-57. doi: http://dx.doi. org/10.1590/2176-9451.19.4.136-157.sar

3. Bhuvaneswaran M. Principles of smile design. J Conserv Dent. 2010; 13 (4): 225. doi: 10.4103/0972-0707.73387

4. Havens DC, McNamara Jr JA, Sigler LM, Baccetti T. The role of the posed smile in overall facial esthetics. Angle Orthod. 2010; 80 (2), 322-8. doi: 10.2319/040409-194.1

5. Van der Geld P, Oosterveld P, Bergé SJ, Kuijpers-Jagtman AM. Tooth display and lip position during spontaneous and posed smiling in adults. Acta Odontol Scand. 2008; 66 (4), 207-13. doi: 10.1080/00016350802060617

6. Krishnan V, Daniel ST, Lazar D, Asok A. Characterization of posed smile by using visual analog scale, smile arc, buccal corridor measures, and modified smile index. Am J Orthod Dentofacial Orthop. 2008; 133 (4), 515-23. doi: 10.1016/j.ajodo.2006.04.046

7. Fradeani M. Evaluation of dentolabial parameters as part of a comprehensive esthetic analysis. Eur J Esthet Dent. 2006; 1: 62-9.

8. Van der Geld P, Oosterveld P, Van Heck G, KuijpersJagtman AM. Smile attractiveness. Self-perception and influence on personality. Angle Orthod. 2007; 77: 759-65. doi: 10.2319/082606-349

9. Mahn E, Sampaio CS, Da Silva BP, Stanley K, Valdés AM, Gutiérrez J, et al. Comparing the use of static versus dynamic images to evaluate a smile. J Prosthet Dent. 2019. doi. org/10.1016/j.prosdent.2019.02.023

10. Cruz GC, Fernández López A, Rodríguez FM. Association between the smile arc and buccal corridors with the facial biotype in subjects with normocclusion. Revista Mexicana de Ortodoncia. 2015; 3 (1): 8-12. doi.org/10.1016/j.rmo.2016.03.003

11. Chirivella P, Singaraju GS, Mandava P, Reddy VK, Neravati JK, George SA. Comparison of the effect of labiolingual inclination and anteroposterior position of maxillary incisors on esthetic profile in three different facial patterns. J Orthod Sci. 2017; 6 (1): 1. doi: $10.4103 / 2278-0203.197387$
Fuente de financiamiento: Autofinanciado.

Conflictos de intereses: El autor declara no tener conflicto de intereses de ningún tipo.

12. Gregoret J, Tuber E, Escobar L, Matos da Fonseca A. Ortodoncia y cirugía ortognática. Diagnóstico y planificación. Barcelona: Publicaciones Médicas Espaxs; 1997.

13. Dawson PE. Determining the determinants of occlusion. Int J Periodontics Restorative Dent. 1983; 3: 8-21.

14. Camara CA. Esthetics in Orthodontics: six horizontal smile lines. Dental Press J Orthod. 2010; 15 (1): 118-31. doi.org/10.1590/ S2176-94512010000100014

15. Mayoral J, Mayoral G. Ortodoncia. Principios fundamentales y práctica. 6.a ed. Barcelona: Labor; 1990.

16. Sabri R. The eight components of a balanced smile. J Clin Orthod. 2005; 39 (3), 155-67.

17. Posada LE, Rúa AR, Bustamante AG, Villa PV. Estudio descriptivo de los rasgos dentales y faciales en varios pacientes de diferentes clínicas de la ciudad de Medellín. Revista CES Odontología. 2003; 16 (1): 13-20.

18. Tjan AH, Miller GD. Some esthetic factors in a smile.J Prosthet Dent. 1984; 51 (1): 24-8. doi: 10.1016/s0022-3913(84)80097-9

19. Zhang YL, Le D, Hu WJ, Zhang H, Liang LZ, Chung KH, Cao ZQ.Assessment of dynamic smile and gingival contour in young Chinese people. Int Dent J. 2015; 65 (4): 182-7. doi: 10.1111/ idj.12174

20. Hwang HS, Kim WS, McNamara JA Jr. Ethnic differences in the soft tissue profile of Korean and EuropeanAmerican adults with normal occlusions and wellbalanced faces. Angle Orthod. 2002; 72 (1): 72-80. doi: 10.1043/0003-3219(2002)072<0072:EDITST>2.0.CO;2

21. Köseoğlu M, Özdemir H, Bayındır F. The evaluation of different smile parameters in the Turkish population. Int Dent Res 2018;8(1):1-6. doi: 10.5577/intdentres.2018.vol8.no1.1

22. Siddiqui N, Tandon P, Singh A, Haryani J. Dynamic smile evaluation in different skeletal patterns. Angle Orthod. 2016; 86 (6): 1019-25. doi: 10.2319/012616-69.1

23. Johnson PF. Racial norms: Esthetic and prosthodontic implications. J Prosthet Dent. 1992; 67 (4): 502-8. doi. org/10.1016/0022-3913(92)90081-k 